\title{
Technological Progress, Obsolescence and Depreciation
}

R. Boucekkine, F. del Rio and B. Martinez

Discussion Paper 2006-15

\section{Département des Sciences Économiques de l'Université catholique de Louvain}




\title{
Technological progress, obsolescence and depreciation*
}

\author{
Raouf Boucekkine $^{\dagger} \quad$ Fernando del Río Blanca Martinez $^{\S}$
}

March 16, 2006

\begin{abstract}
We construct a vintage capital model à la Whelan (2002) with both exogenous embodied and disembodied technical progress, and variable utilization of each vintage. The lifetime of capital goods is endogenous and it relies on the associated maintenance costs. We study the properties of the balanced growth paths. First, we show that the lifetime of capital is an increasing (resp. decreasing) function of the rate of disembodied (resp. embodied) technical progress. Second, we show that both the use-related depreciation rate and the scrapping rate increase when embodied technical progress accelerates. However, the latter drops when disembodied technical progress accelerates while the former remains unaffected. A key feature of our model is that the age-related depreciation rate does depend on the obsolescence rate in sharp contrast to the neoclassical model.
\end{abstract}

Keywords: Vintage capital, operation costs, embodied technical progress, age-related depreciation, obsolescence

Journal of Economic Literature: E22, E32, O40.

\footnotetext{
*We would like to thank Omar Licandro, Luis Puch and Ramon Ruiz-Tamarit for stimulating and useful comments. R. Boucekkine acknowledges the support of the Belgian research programmes PAI P4/01 and ARC 03/08-302. F. del Río acknowledges the financial support from the Spanish CICYT Project SEJ200404579. B. Martínez acknowledges the financial support of the Spanish Project SEJ2005-02829/ECON.

†Université catholique de Louvain. boucekkine@ires.ucl.ac.be

${ }_{\ddagger}^{\ddagger}$ Corresponding author. Departamento de Fundamentos da Análise Económica, Facultade CC. Económicas e Empresariais, Avda. Xoan XXIII s/n, Universidade de Santiago de Compostela, Santiago de Compostela, Spain. aedelrio@usc.es

§Universidad Complutense de Madrid. blmartin@ccee.ucm.es
} 


\section{Introduction}

The topic of replacement investment and capital depreciation has always been a concern for economic theorists and practitioners. This concern comes principally from the feeling that the assumption of a constant depreciation rate (and therefore an assumption of a constant replacement investment to capital ratio) is barely incorrect. This assumption is for example strongly challenged by Feldstein and Rotschild (1974) and Nickell (1975) in pioneering theoretical contributions. An early empirical assessment of this issue is due to Griliches (1960) who studied the replacement of farm tractors and proposed a way to measure capital depreciation in this context.

An obvious alternative to the constant depreciation rate assumption is the well-known depreciation-in-use assumption. Typically, capital depreciation is varying over time depending for example on the pace of economic activity. A higher level of economic activity is generally associated with a higher rate of capital utilization, which accelerates the depreciation of capital. This endogenous view of depreciation, often referred to as the depreciation in use hypothesis, has been put forward by Epstein and Denny (1980) and Bischoff and Kokkelenberg (1987). ${ }^{1} \quad$ While this approach is certainly worthwhile, it does not seem to be completely satisfactory: It assigns a residual role to capital depreciation, and it is quite mechanically computed from the rate of capital utilization optimal paths once the optimal investment plan of the representative firms is characterized.

In this paper we develop a theory of capital depreciation based in the existence of maintenance costs. McGrattan and Schmitz (1999) have highlighted the quantitative importance

\footnotetext{
${ }^{1}$ Real business cycles models incorporating depreciation in use have been also built up and simulated in order to assess the cyclical implications of this hypothesis. Among others, the seminal contributions of Greenwood, Hercowitz and Huffman (1988) and Burnside and Eichenbaum (1996).
} 
of the maintenance and repair costs. These authors have found in Canada for the period 1961-1993, that up to $6 \%$ of gross national product was devoted to repair and maintenance activities, which is approximately half expenditure made on the acquisition of new capital goods. At the firm level, there exists a large microeconomic literature on the importance of maintenance and repair of cars (see for example, Hamilton and Macauley, 1998). At this level, depreciation is no longer a residual variable: It is an important control variable, as important as investment itself, and the rate of utilization of capital. Typically, firms choose an optimal operation and maintenance policy together with their investment plan (see for example Boucekkine and Ruiz-Tamarit, 2003). Apart from these quite obvious microeconomic considerations, there is now a growing view that depreciation is a crucial and nontrivial economic phenomenon when accounting for the economic performances at the aggregate level. An early contribution highlighting the role of replacement investment is in Gylafson and Zoega (2001): using a World Bank data, they show that average depreciation of fixed capital during the period 1970-1998, measured as a proportion of GDP, is directly related to initial GNP per capita across 85 countries as well as to the average growth rate of output per capita.

Our paper builds on Whelan's (2002) contribution. As this author, we consider a vintage capital model with endogenous capital goods' lifetime. A particular vintage is scrapped when its profitability is not enough to compensate the corresponding maintenance costs. Whelan assumes that this maintenance cost is fixed. In contrast to Whelan, in our model the maintenance costs are twofold, a fixed and a variable cost. The variable cost depends on an indicator of the utilization of the vintages. No endogenous utilization indicator is considered by Whelan, and we believe it plays an important role in both the investment and 
maintenance decisions, and in the resulting depreciation. Thanks to this difference, we are able to distinguish between an endogenous use-related depreciation rate (depending mostly on the variation in the utilization variable) and an endogenous scrapping rate (depending mostly on the lifetime of capital goods). It is worth pointing out here that our concept of use-related depreciation is due to the decline of the optimal utilization of capital when new and more productive capital goods arrive, and old capital goods become obsolete. Therefore, the use-related depreciation is linked to the age of capital, and it is substantially different from the depreciation in use framework described above, which merely reflects the increasing deterioration of capital goods (independently of their age) in times of larger rates of capital utilization.

In this framework, we study the relationship between the rates of embodied and disembodied technical progress and the depreciation rate of capital. First, it is analytically shown that the lifetime of capital is an increasing (resp. decreasing) function of the rate of disembodied (resp. embodied) technical progress. Second, we show that both the use-related depreciation rate and the scrapping rate increase when embodied technical progress (or the obsolescence rate) accelerates. In contrast, the latter drops when disembodied technical progress accelerates while the former remains unaffected. In contrast to the Whelan's contribution, which is mainly empirical, we produce a full analytical characterization, which is far from a simple task as it will appear clearly along the way. The reason why the analytical characterization is quite hard in this kind of models is mentioned in Boucekkine et al. (1998): A technological acceleration induces on one hand an incentive to scrap the machines earlier in order to profit from the increasing efficiency of new vintages, but on the other hand, a rising rate of technological progress pushes the interest rate upward, ${ }^{2}$ which tends to reduce the profitability

\footnotetext{
${ }^{2}$ This is a standard property in optimal growth models with exogenous technical progress, it is typically
} 
of investment and requires a bigger lifetime service of equipment in order to equalize the marginal profitability and marginal cost of investment. This ambiguity gives rise to a real analytical problem.

Our framework allows us to re-examine several empirical issues, from Whelan's productivity analysis focus to Geske, Ramey and Shapiro's 2004 paper on the decomposition of nonfinancial user cost of personal computers in the recent years in the US. Section 2 is precisely devoted to highlight these issues and to give a flavor of the achievements of our work in this respect. Section 3 presents the model, and identifies neatly the corresponding use-related depreciation rate and the scrapping rate. Section 4 studies the balanced growth paths of the model, including the existence-uniqueness issue. Section 5 is devoted to characterize how the lifetime of capital goods, the rate of use-related depreciation and the rate of scrapping move under embodied Vs disembodied technical progress accelerations. In this section, we discuss the relationship between the age-related depreciation rate and the obsolescence rate. Section 6 concludes.

\section{Some key preliminary empirical observations}

\section{Some stylized facts on depreciation}

On US data, the available evidence seems to suggest on one hand that the depreciation rate of capital has not been constant in the recent period, and on the other hand, that it was quite reactive to technological evolutions. Indeed, using a data on capital depreciation built up by BEA, it can be neatly shown that the depreciation rate of US non-residential private fixed equipment and software has increased from $1960 .^{3}$ This increase in the depreciation reflected in the so-called Fisher equation.

\footnotetext{
${ }^{3}$ The relative price of equipment is the ratio "NIPA price index of private nonresidential equipment
} 
rate has been accompanied by an increase in the decline rate of the NIPA relative price of non residential private fixed equipment and software (see Figure 1). The relative price of investment can be seen as a proxy of the embodied technical progress (see Greenwood, Hercowitz and Krusell, 1997). Therefore, Figure 1 suggests a positive relationship between the depreciation rate of capital and the rate of embodied technical progress.

This fundamental property can also be recovered using a cross-section analysis based on Table 1, which is reported in the Appendix. This table summarizes the magnitudes currently considered by BEA: It gives the depreciation rate, the service lifetime in years, and the decline rate of the relative price of equipment and software by equipment types. Figure 2 and 3 illustrate the main regularities entailed in Table 1. Figure 2 shows that there is a positive correlation between the depreciation rates of the categories of equipment and software used by $\mathrm{BEA}$ and the decline rate of their corresponding relative prices. Analogously Figure 3 shows that the service lifetime of the different types of non-residential private equipment and software is negatively correlated with the decline rate of their relative prices.

\section{Measuring capital depreciation and productivity growth}

As pointed out by Fraumeni (1997), "There are two possible sources of the price change: the first being a change in the price of an asset because it has aged and the second being a change in the price of an asset because it is a different time period". The first change rate can be referred to as age-related depreciation rate, and the second one may be called time-related and software" over "NIPA price index of non-durables consumption and services". The depreciation rate is calculated as follows. Both the chain-type quantity index for the net stock of private nonresidential equipment and software, and the chain-type quantity index for depreciation of private nonresidential equipment and software are multiplied by their respective historical cost in year 1996. The depreciation rate is calculated dividing the chain-dollar series of depreciation by the chain-dollar series of the net stock of equipment and software. 
depreciation rate. ${ }^{4}$ The neoclassical growth model assumes an exogenous and constant agerelated depreciation rate of capital, ${ }^{5}$ and time-related depreciation rate of capital is equal to the obsolescence rate, which is in turn equal to the rate of embodied technical progress. Usually the sum of both rates is called the economic depreciation rate.

BEA uses used-assets prices unadjusted for quality to estimate the depreciation rates of the different types of capital goods. Therefore, the BEA depreciation rates include both types of depreciation (age-related and time-related). So it is little surprising that capital economic depreciation, as measured by BEA, increases when the relative price of new capital equipment decreases at a faster rate, thus speeding up obsolescence. Our analysis allows for a deeper analysis of this phenomenon. In particular, we point out that obsolescence affects the economic depreciation of capital through additional indirect channels. Indeed, our models predicts that an acceleration in embodied technical progress induces a faster decline in the capital utilization of aging capital goods and a shorter capital lifetime. Therefore, in contrast to the neoclassical growth model, the age-related depreciation rate depends on the obsolescence rate, which seems rather consistent with the data as we shall see in Section 5. If this effect of the obsolescence rate on the age-related depreciation rate is not taken into account, then the growth rate of capital accumulation can be overestimated and this may yield misleading estimation of total factor productivity growth. Whelan (2002) has clearly illustrated this point when measuring the usage effect of computers on US productivity. ${ }^{6}$ Moreover, we shall also show that this dependence of the age-related depreciation rate on the obsolescence rate allows to explain the Geske, Ramey and Shapiro finding. Re-

\footnotetext{
${ }^{4}$ The former is called depreciation by Fraumeni (1997), and the latter is called revaluation by the same author.

${ }^{5}$ It is often called the physical depreciation rate.

${ }^{6}$ More recently, Musso (2004) obtains the same kind of results when simulating a computable general equilibrium model à la Whelan.
} 
cently, Geske, Ramey and Shapiro (2004) have studied the decomposition of non-financial user cost of personal computers in the recent years in the US. They explicitly distinguish between obsolescence and age-related depreciation (or deterioration). Applied to the recent US experience, they find that the role of age-related depreciation is quite negligible while obsolescence turns out to be a major source of change in the user cost of computers. Our framework gives a neat rationale to this finding.

\section{Some critical implications of a finite capital lifetime}

It is worth pointing out that BEA assumes constant depreciation rates for all categories of non-residential private equipment and software except computing equipment and autos. ${ }^{7}$ As Whelan (2002) has already mentioned, the depreciation rates for computing equipment have actually increased over time. Our model predicts that if the lifetime of the capital goods is finite, then their depreciation schedules cannot be geometric (or equivalently, depreciation rates cannot be constant). Moreover, we also show that the difference between the true depreciation schedule and a geometric depreciation schedule is higher as the lifetime of a capital good gets shorter, which use to happen when embodied technical progress accelerates. In such a circumstance, the assumption of a constant depreciation rate becomes even less appropriate.

We finally point at another critical implication of the finite capital lifetime property. BEA uses used-asset prices to estimate the depreciation rate of the different types of capital, and these estimated depreciation rates are later employed to build the stocks of capital. This is of course correct under the assumptions of the standard neoclassical growth model. However, if

\footnotetext{
${ }^{7}$ Therefore, the increase of the depreciation rate of non residential private equipment and software is mainly due to a composition effect, with the sharply rising weight of computers and autos in the stock of non-residential private equipment and software.
} 
the lifetime of capital is finite, then the decline rates of used-asset prices need not be equal to the depreciation rates of the corresponding capital stocks. We show this neatly in Section 5 . The rationale behind this is pretty clear: if the lifetime of capital is finite, the depreciation due to scrapping does depend on the amount of capital invested in the past, and not only on the price change of a unit of capital.

\section{The model}

New plants are built in each period. Each plant at time $z$ is built with a unit of capital. The production function at time $t$ of a plant built at time $z$ (hereafter, a plant of vintage $z$ ) is Cobb-Douglas,

$$
Y_{z, t}=A \mathrm{e}^{\gamma t}\left(\mathrm{e}^{\lambda z} U_{z, t}\right)^{\alpha} L_{z, t}^{1-\alpha}
$$

where $0<\alpha<1, Y_{z, t}$ is output of a plant of vintage $z$ at time $t, L_{z, t}$ is labor employed in an plant of vintage $z$ at time $t, A>0$ is the level of disembodied technical knowledge which grows at the rate $\gamma \geq 0, \mathrm{e}^{\lambda z}$ is the state of embodied technical knowledge in vintage $z$ and $U_{z, t}$ is an index of utilization of capital of the plant of vintage $z$ at time $t$.

The maintenance cost of vintage $z$ at time $t$, say $M_{z, t}$, depends mainly on its utilization. More specifically, we assume that the maintenance costs function, $M_{z, t}\left(U_{z, t}\right)$, does satisfy the following properties: (i) it is an increasing and convex function of utilization: $M^{\prime}(U)>0$, $M^{\prime \prime}(U)>0$ for all positive $U$, (ii) $M(0)>0$ which reflects the existence of a support cost. Hereafter, the following function of maintenance costs is assumed in order to get analytical results:

$$
M_{z, t}\left(U_{z, t}\right)=\beta \mathrm{e}^{\chi(t-z)} U_{z, t}^{\mu}+\eta
$$


where $\beta>0, \eta>0$ and $\mu>1$. The following parametric assumption must be hold:

$$
\chi>0 \text { and/or } \lambda>0
$$

The existence of the fixed cost, $\eta>0$, together with assumption (2) are needed to have a finite optimal lifetime of the vintage, as it will be clear later. Note that we assume that the maintenance costs can increase over time. Among other acceptable reasons, this might be attributed to the fact that old machines become less compatible with new ones.

The optimization problem of a plant

Profit of vintage $z$ at time $t$ are:

$$
\pi_{z, t}=A \mathrm{e}^{\gamma t}\left(\mathrm{e}^{\lambda z} U_{z, t}\right)^{\alpha} L_{z, t}^{1-\alpha}-W_{t} L_{z, t}-\beta \mathrm{e}^{\chi(t-z)} U_{z, t}^{\mu}-\eta
$$

where $W_{t}$ is wage at time $t$. Vintage $z$ chooses $U_{z, t}$ and $L_{z, t}$ in order to maximize its profits:

$$
\begin{gathered}
W_{t}=(1-\alpha) A \mathrm{e}^{(1-\alpha) g t}\left(\mathrm{e}^{-\lambda(t-z)} U_{z, t}\right)^{\alpha} L_{z, t}^{-\alpha}, \\
\alpha A \mathrm{e}^{(1-\alpha) g t} \mathrm{e}^{-\alpha \lambda(t-z)} U_{z, t}^{\alpha-1} L_{z, t}^{1-\alpha}=\beta \mu \mathrm{e}^{\chi(t-z)} U_{z, t}^{\mu-1},
\end{gathered}
$$

where $g=\frac{\alpha \lambda+\gamma}{1-\alpha}$. Equation (4) states that the marginal productivity of labor equals wage in each period. Equation (5) states that the optimal utilization of a vintage is such that its marginal productivity equals its marginal cost. From equation (4) it follows that marginal productivity of labor is equal across vintages and then:

$$
L_{z, t}=\frac{U_{z, t}}{U_{t, t}} e^{-\lambda(t-z)} L_{t, t}
$$

which states that employment in a plant of age $t-z$ equals employment in a new plant times its relative utilization. Evaluating (4) in $z=t$, it follows that employment in a new plant is given by:

$$
L_{t, t}=\left(\frac{W_{t}}{A(1-\alpha)} \mathrm{e}^{-g t}\right)^{-\frac{1}{\alpha}} \mathrm{e}^{-g t} U_{t, t} .
$$


From equations (4) and (5), and after a little some straightforward algebra, one might conclude that capital utilization of a vintage is a decreasing function of its age:

$$
U_{z, t}=U_{t, t} \mathrm{e}^{-\delta(t-z)}
$$

where $\delta=\frac{\chi+\lambda}{\mu-1}$ and the initial utilization of a new plant, $U_{t, t}$, is a decreasing function of wage,

$$
U_{t, t}=\left(\frac{\alpha A}{\mu \beta}\right)^{\frac{1}{\mu-1}}\left(\frac{W_{t}}{A(1-\alpha)} \mathrm{e}^{-g t}\right)^{-\frac{\theta}{\mu \alpha}}
$$

with $\theta=\frac{\mu(1-\alpha)}{\mu-1}$. Both utilization and employment of the plant fall when it becomes older. The decline rates of employment and utilization are increasing functions of the rate of embodied technical progress. This is the obsolescence effect of embodied technical progress.

Substituting (4) and (5) into (3) yields: $\pi_{z, t}=\frac{\mu-1}{\mu} \frac{\alpha}{1-\alpha} W_{t} L_{z, t} \mathrm{e}^{-(\delta+\lambda)(t-z)}-\eta$. And substituting from $(6),(7),(8)$ and (9) into previous equation, one finally gets:

$$
\pi_{z, t}=\Omega\left(\frac{W_{t}}{A(1-\alpha)} \mathrm{e}^{-g t}\right)^{-\frac{\theta}{\alpha}} \mathrm{e}^{-(\delta+\lambda)(t-z)}-\eta .
$$

where $\Omega=\frac{\mu-1}{\mu} \alpha A\left(\frac{\alpha A}{\beta \mu}\right)^{\frac{1}{\mu-1}}$. It is clear from the previous equation that when the plant becomes older, its profits go down because its utilization and employment decay due to obsolescence.

A vintage is scrapped in period $z+J_{z}$ when it becomes unprofitable, $\pi_{z, z+J_{z}}=0$,

$$
\Omega \mathrm{e}^{-(\delta+\lambda) J_{z}}\left(\frac{W_{z+J_{z}}}{A(1-\alpha)} \mathrm{e}^{-g\left(z+J_{z}\right)}\right)^{-\frac{\theta}{\alpha}}=\eta .
$$

And it must be hold that the lifetime of vintage $z$ equals the scrapping time at time $z+J_{z}$ :

$$
J_{z}=T_{z+J_{z}}
$$

There is free entry and exit of plants and the number of plants of a vintage is determined 
by a zero profits condition:

$$
\int_{z}^{z+J_{z}} \mathrm{e}^{-\int_{z}^{t} r_{s} d s}\left(\Omega \mathrm{e}^{-(\delta+\lambda)(t-z)}\left(\frac{W_{t}}{A(1-\alpha)} \mathrm{e}^{-g t}\right)^{-\frac{\theta}{\alpha}}-\eta\right) d t=1,
$$

where $r_{s}$ is the interest rate at time $s$, and which states that the discounted sum of profits of a plant must be equal to the cost of a unit of capital.

\section{Aggregating}

The aggregate production at time $t, Y_{t}$, is the sum of output of all plants surviving at time $t$

$$
Y_{t}=\int_{t-T_{t}}^{t} I_{z} A \mathrm{e}^{\gamma t}\left(\mathrm{e}^{\lambda z} U_{z, t}\right)^{\alpha} L_{z, t}^{1-\alpha} d z
$$

where $T_{t}$ is the age of the oldest plants still in use at time $t$ and $I_{z}$ is the number of plants of vintage $z$ (and aggregate investment at time $z$ ). Aggregate employment is the sum of employment of all plants surviving at time $t$,

$$
L_{t}=\int_{t-T_{t}}^{t} I_{z} L_{z, t} d z
$$

Substituting from (4) into (15) after a little of algebra yields:

$$
W_{t}=(1-\alpha) A \mathrm{e}^{(1-\alpha) g t} K_{t}^{\alpha} L_{t}^{-\alpha}
$$

where

$$
K_{t}=\int_{t-T_{t}}^{t} U_{z, t} I_{z} \mathrm{e}^{\lambda(z-t)} d z
$$

is the replacement value of capital, and it is the theoretical counterpart of the NIPA realcost net stock of capital (see BEA(2003)). ${ }^{8}$ Differentiating previous equation and after some algebra, we obtain the evolution law of the replacement value of capital,

$$
\frac{\mathrm{d} K_{t}}{\mathrm{~d} t}=U_{t, t} I_{t}-\left(\delta_{t}+\xi_{t}+\lambda\right) K_{t},
$$

\footnotetext{
${ }^{8}$ The quality-unadjusted relative price of investment is 1 for all $t$, but the quality-adjusted relative price of investment at time $t$ is $\mathrm{e}^{-\lambda t}$.
} 
where

$$
\delta_{t}=\frac{-1}{K_{t}} \int_{t-T_{t}}^{t} e^{\lambda(z-t)} U_{z, t} I_{z} \frac{\mathrm{d} U_{z, t}}{\mathrm{~d} t} \frac{1}{U_{z, t}} d z
$$

is the use-related depreciation rate, which captures the decline of utilization of capital when its age increases, while

$$
\xi_{t}=\left(1-\frac{\mathrm{d} T_{t}}{\mathrm{~d} t}\right) \frac{\mathrm{e}^{-\lambda T_{t}} U_{t-T_{t}, t} I_{t-T_{t}}}{K_{t}}
$$

is the fraction of capital scrapped at time $t$ because it is not profitable, and it is called the scrapping rate. The obsolescence rate is $\lambda$, which is equal to the rate of embodied technical progress. ${ }^{9}$ Under our Cobb-Douglas assumption aggregate output is a function of aggregate capital and aggregate employment:

$$
Y_{t}=A e^{(1-\alpha) g t} K_{t}^{\alpha} L_{t}^{1-\alpha}
$$

Equation (21) has been obtained substituting (4) into (14) and using (16).

Closing the model

The aggregate operation cost is given by:

$$
M_{t}=\int_{t-T_{t}}^{t} I_{z}\left(\beta \mathrm{e}^{\chi(t-z)} U_{z, t}^{\mu}+\eta\right) d z
$$

which corresponds actually to fraction of aggregate output plus the sum of surviving investments times the fixed cost $\eta$ :

$$
M_{t}=\frac{\alpha}{\mu} Y_{t}+\eta \int_{t-T_{t}}^{t} I_{z} d z
$$

Equation (23) is obtained by substituting (5) into (22).

The representative household is composed of $L_{t}$ individuals at time $t$. At any period $t$, $L_{t}$ grows at the constant rate $n \geq 0$. The utility function of the representative household

\footnotetext{
${ }^{9}$ It is also equal to the decline rate of the quality-adjusted relative price of investment.
} 
is $U_{t}=\int_{0}^{\infty} L_{t} e^{-\rho t} \frac{C_{t}^{1-\sigma}}{1-\sigma} d t$, where $C_{t}$ is consumption per capita, $\sigma>0$ is the intertemporal elasticity of substitution and $\rho>0$ is the discounted parameter The Euler condition of the maximization problem of the representative household is:

$$
\frac{\frac{\mathrm{d} C_{t}}{\mathrm{~d} t}}{C_{t}}=\frac{1}{\sigma}\left(r_{t}-\rho\right)
$$

Finally, the resource constraint is

$$
Y_{t}=C_{t} L_{t}+I_{t}+M_{t}
$$

which states that output equals the sum of consumption, investment and operation costs.

\section{Balanced Growth Path}

In this section, we study the existence and uniqueness of balanced growth paths. We shall define a balanced growth path (BGP hereafter) as follows:

Definition 1 Along a BGP, the lifetime of vintages, $T=J$, is constant. Consumption per capita, production per capita, investment per capita and operation costs per capita grow at the same constant (steady state) rate $g$.

We now study whether our model admits such a solution. As usual in this class of models (see for example, Boucekkine et al., 1998), this question turns out to be whether the BGP restrictions stated above imply a unique solution for the lifetime variable, the stationary levels of the other variables being trivially computable when the value of capital's lifetime is available. Before moving to this mathematical issue, we will characterize the main economic properties of the BGP of our model.

First of all, notice that under our definition, the capital stock grows at the rate $g$, which implies that both the use-related depreciation rate and the scrapping rate are constant 
along a BGP. The steady state growth rate $g$ can be therefore readily computed from the Cobb-Douglas production function (21), $g=\frac{\gamma+\lambda \alpha}{1-\alpha}$. Hereafter a lower case, $x$, denotes the corresponding variable denoted by an upper case detrended and in terms per capita. We shall impose the following condition: $(1-\sigma) g<\rho$, which guarantees that the intertemporal utility is bounded. We now look at some properties of the per vintage distributions in the BGPs.

\section{Utilization per vintage}

Along a BGP endogenous utilization of a vintage evolves according to:

$$
U_{z, t}=U_{0} \mathrm{e}^{-\delta(t-z)} \text { for all } t-z \in[0, T]
$$

where

$$
U_{0}=U_{t, t}=\left(\frac{\alpha A}{\beta \mu}\right)^{\frac{1}{\mu-1}} k^{-\frac{1}{\mu} \theta},
$$

is the initial utilization of a vintage and it is constant along a BGP because the aggregate capital-labor ratio grows at the constant rate $g$ along a BGP. Equation (26) follows from substituting (16) into (9). Equation (25) shows that the utilization of a vintage decreases

with its age at the rate $\delta=\frac{\lambda+\chi}{\mu-1}$ due to obsolescence: when a vintage goes away from the technological frontier, the firm optimally decides to devote less resources to operate it.

\section{The long run depreciation rates}

The use-related depreciation rate is given by equation (19). As explained just above, the decline rate of utilization of a vintage is constant in the BGP and equal to

$$
\delta=\frac{\lambda+\chi}{\mu-1}
$$

The use-related depreciation rate equals the rate at which utilization of capital declines when it becomes older. The scrapping rate is given by equation (20), which along a BGP is 
constant and given by

$$
\xi=U_{0} \frac{i}{k} e^{-(\delta+g+n+\lambda) T}
$$

It follows from the evolution law of capital (18) that along a BGP investment is such that capital per capita grows at the constant rate $\lambda+g$,

$$
\frac{U_{0} i}{k}=(\delta+\xi+n+\lambda+g)
$$

Using (28) and (29), the scrapping rate can be written as:

$$
\xi=\frac{\delta+n+\lambda+g}{\mathrm{e}^{(\delta+n+\lambda+g) T}-1}
$$

Characterizing a $B G P$

A BGP is characterized by the following set of equations together with (26), (27), (29) and $(30)$ :

$$
\begin{gathered}
w=(1-\alpha) A k^{\alpha} \\
\Omega \mathrm{e}^{-(\delta+\lambda) T} k^{-\theta}=\eta \\
\Omega k^{-\theta}-\eta=r+\delta+\lambda+\frac{(\delta+\lambda) \eta}{r}\left(1-\mathrm{e}^{-r T}\right) \\
m=\frac{\alpha}{\mu} A k^{\alpha}+\frac{\eta}{g} i\left(1-\mathrm{e}^{-(g+n) T}\right) \\
r=\sigma g+\rho \\
A k^{\alpha}=c+i+m .
\end{gathered}
$$

Equation (31) states that marginal productivity of labor equals wage. Equation (32) is the scrapping condition, and it states that a vintage will be scrapped when its profitability is zero. Equation (33) states that the marginal productivity of capital equals its user cost, and it has been obtained, using (31), by differentiating the zero profits condition (13) under the 
assumptions characterizing a BGP. The user cost of capital is the sum of the interest rate, $r$, the use-related depreciation rate, $\delta$, the obsolescence rate, $\lambda$, and a last term depending on the fixed operation cost, $\frac{(\delta+\lambda) \eta}{r}\left(1-\mathrm{e}^{-r T}\right) \cdot{ }^{10}$ Equation (34) gives the aggregate operation costs as a function of output, investment and the optimal lifetime of capital. Equations (35) and (36) show the Euler condition and the resource constraint.

The steady state value for the lifetime of capital

The following proposition states that there is a lifetime of capital strictly positive and it is unique.

Proposition $2 T>0$ exists and is unique.

Proof: Using (31), (32) and (35) the zero profits condition (13) along a BGP becomes:

$$
\int_{0}^{T}\left(\mathrm{e}^{-(\delta+\lambda)(a-T)}-1\right) \mathrm{e}^{-(\sigma g+\rho) a} d a=\frac{1}{\eta}
$$

The left hand side of (37) is a continuous and strictly increasing function of $T$, and its limit when $T$ goes to zero is 0 and when $T$ goes to infinity is $\infty$. The right hand side is a positive constant. Proposition 1 follows from the theorem of the intermediate value.

Hence, our model admits a unique BGP. We are now ready to make our point and in particular to study how the age-related and scrapping rates move under exogenous technological accelerations. The next section is therefore exclusively devoted to the analysis of the comparative statics of the depreciation variables (including scrapping time) with respect to the rates of embodied and disembodied technological progress. Some more comparative statics are added to better assess the properties of the BGP of our model.

\footnotetext{
${ }^{10}$ The scrapping costs are not in equation (33) because the optimal choice of $U_{z, t}$ implies that profitability of a vintage is zero when it is $T$ years old.
} 


\section{Technical progress and depreciation}

Since $T$ is by construction a crucial determinant of depreciation, we start with the former variable. We then study how the two forms of technical progress affect the rates of use-related depreciation and scrapping.

\subsection{Embodied Vs disembodied technical progress and the lifetime of capital}

The following proposition states some properties of static comparative of the lifetime of capital:

Proposition 3 The lifetime of capital is an increasing function of $\sigma, \rho$ and $\mu$, a decreasing function of $\chi$ and $\eta$, and it does not depend on $A, \beta$ and $n$.

Proof: Equation (37) does not depend on $\beta, A$ and $n$, therefore $\frac{\partial T}{\partial \beta}=0, \frac{\partial T}{\partial A}=0$, $\frac{\partial T}{\partial n}=0$. From (37) follows that any parametric change increasing (resp. decreasing) $B(a)=$ $\left(e^{(\delta+\lambda)(T-a)}-1\right) \mathrm{e}^{-(\sigma g+\rho) a}$ for all for all $a \in[0, T)$ implies a lower $T$. Differentiating $B(a)$,

$$
\begin{gathered}
\frac{\partial B}{\partial(\delta+\lambda)}=(T-a) e^{(\delta+\lambda)(T-a)} e^{-(\sigma g+\rho) a}>0, \\
\frac{\partial B}{\partial \sigma}=-g a\left(e^{(\delta+\lambda)(T-a)}-1\right) e^{-(\sigma g+\rho) a}<0, \\
\frac{\partial B}{\partial \rho}=-a\left(e^{(\delta+\lambda)(T-a)}-1\right) e^{-(\sigma g+\rho) a}<0,
\end{gathered}
$$

for all $a \in[0, T)$, and $\delta$ is a decreasing function of $\mu$ and an increasing function of $\chi$. Then it follows that $\frac{\partial T}{\partial \mu}>0, \frac{\partial T}{\partial \chi}<0, \frac{\partial T}{\partial \sigma}>0$ and $\frac{\partial T}{\partial \rho}>0$. The right hand side of (37) is decreasing with $\eta$, then it follows from (37) that $\frac{\partial T}{\partial \eta}<0$.

Since the population growth rate does not affect the marginal profitability of vintages, it does not influence the lifetime of capital. The disembodied level of productivity, $A$, and the level 
of the variable operation costs given the utilization level, $\beta$, do not show up in the stationary value for the lifetime of capital because changes in these parameters have two opposite effects on this variable, which just offset. Actually, an increase in $A$ (resp. a decrease of $\beta$ ) rises the marginal profitability of any vintage, which tends to increase $T$, but this higher profitability stimulates investment, which ultimately induces a drop in the marginal profitability of the vintage because wages increase, and hence a lower $T$. Both effects just offset.

A lower elasticity of the variable operation costs with respect to the utilization level, $\mu$, or a higher growth rate of the variable operation costs with the age of the vintage, $\chi$, both imply a lower lifetime of capital. The reason is that both parametric changes accelerate the decline of the utilization of capital with the age of the vintage. A higher $\sigma$ or $\rho$ implies a higher interest rate which reduces the present value of profits, and requires a higher $T$ to equalize the marginal profitability and the marginal cost of investment and to restore the optimal rule given by equation (37).

We now turn to the analysis of the more important relationship between scrapping and technological progress. The integral equation (37) makes it clear that this relationship might not be easy to characterize. We first state the easier results.

Proposition 4 The lifetime of capital is an increasing function of the rate of disembodied technical progress, $\gamma$. Moreover, the product $\lambda T$ is an increasing function of the rate of embodied technical progress, $\lambda$.

Proof: To ease the exposition, we shall call $F(T, \lambda)$ the integral function appearing in the left hand side of equation (37). Since $g=\frac{\gamma+\lambda \alpha}{1-\alpha}, g$ is an increasing function of $\gamma$ and $B(a)=\left(\mathrm{e}^{(\delta+\lambda)(T-a)}-1\right) \mathrm{e}^{-(\sigma g+\rho) a}$ is a decreasing function of $\gamma$, then the left hand side of $(37)$ is a decreasing function of $\gamma$ for all $a \in[0, T)$, it follows from (37) that $\frac{\partial T}{\partial \gamma}>0$. Unfortunately, the relationship between $T$ and $\lambda$ is much more complex. However, we can prove that 
the product $\lambda T$ is an increasing function of $\lambda$. Indeed, $\frac{\partial(\lambda T)}{\partial \lambda}=T-\lambda\left(\frac{\partial F}{\partial \lambda} / \frac{\partial F}{\partial T}\right)$, which implies: $\frac{\partial F}{\partial T} \frac{\partial(\lambda T)}{\partial \lambda}=T \frac{\partial F}{\partial T}-\lambda \frac{\partial F}{\partial \lambda}$. Given that $\frac{\partial F}{\partial T}>0, \lambda T$ is increasing with $\lambda$ if and only if $\Delta(T, \lambda)=T \frac{\partial F}{\partial T}-\lambda \frac{\partial F}{\partial \lambda}>0$. Using the exact expressions of the involved partial derivatives of function $F$, we find:

$$
\begin{aligned}
\Delta(T, \lambda)=(\delta+\lambda) e^{(\delta+\lambda) T} & \int_{0}^{T} T e^{-(\sigma g+\rho+\delta+\lambda) a} \mathrm{~d} a-\frac{\lambda \mu}{\mu-1} e^{(\delta+\lambda) T} \int_{0}^{T}(T-a) e^{-(\sigma g+\rho+\delta+\lambda) a} \mathrm{~d} a+ \\
& +\frac{\sigma \lambda \alpha}{1-\alpha} \int_{0}^{T} a e^{-(\sigma g+\rho) a}\left(e^{(\delta+\lambda)(T-a)}-1\right) \mathrm{d} a
\end{aligned}
$$

Now, a quick look at the first two terms of the expression above is sufficient to see that the positivity of $\Delta(T, \lambda)$ is ensured if $\delta+\lambda-\frac{\lambda \mu}{\mu-1}>0$. The latter property is clear because $\delta+\lambda-\frac{\lambda \mu}{\mu-1}=\frac{\chi}{\mu-1}>0$.

An increase in the rate of disembodied technical change $\gamma$ has the same two effects as an increase in $A$ on the lifetime value $T$. As for the parameter $A$, these two effects just offset. A third effect additionally arises: A higher $\gamma$ implies a higher interest rate which reduces the present value of profits. A higher $T$ is needed to equalize the marginal profitability and the marginal cost of investment, so that the optimal rule (37) is re-established. An increase in the embodied technical progress has an ambiguous effect on the lifetime of capital. There are two opposite effects of a change in the rate of embodied technical progress on the lifetime of capital. An increase of $\lambda$ accelerates the decline rate of the vintage utilization and vintage employment (with the age of the vintage), which implies a lower lifetime of capital. However, a rise of $\lambda$ increases the interest rate which reduces the present value of profits, and would require as before a higher $T$ to equalize the marginal profitability and the marginal cost of investment. Whether the first or the second effect dominates is not clear at all.

However, the proposition states that even if $T$ drops under an acceleration in the rate of embodied technical progress, the size of this drop cannot be bigger than the size of the 
acceleration. The next proposition exhibits a sufficient condition under which the lifetime $T$ is indeed a decreasing function of the rate of embodied technical progress. As we shall see afterwards, this should be the case when the parameters of the model take the values usually considered in the literature.

Proposition $5 T$ is a decreasing function of $\lambda$ provided $T \leq \frac{\kappa}{\delta+\lambda}$, where $\kappa=\frac{1-\alpha}{\alpha \sigma} \frac{\mu}{\mu-1}$. A necessary and sufficient condition on the parameters for the latter inequality to hold is: $\frac{1}{\eta} \leq F\left(\frac{\kappa}{\delta+\lambda}, \lambda\right)$.

Proof: The second part of the proposition is a direct consequence of the monotonicity of function $F(T, \lambda)$ with respect to the first argument, and equation (37). The first part can be handled using the fact that function $F(T, \lambda)$ can be rewritten as:

$$
F(T, \lambda)=e^{(\delta+\lambda) T} \int_{0}^{T} \int_{z}^{T}(\delta+\lambda) e^{-r z-(\delta+\lambda) x} \mathrm{~d} x \mathrm{~d} z
$$

where $r=\sigma g+\rho$. Differentiating $F(T, \lambda)$ with respect to $\lambda$, one can readily see that the sign of this derivative entirely depends on the sign of:

$$
\int_{0}^{T} \int_{z}^{T}\left[(\delta+\lambda) \frac{\mu T}{\mu-1}+\frac{\mu}{\mu-1}+(\delta+\lambda)\left(-\frac{\partial r}{\partial \lambda} z-\frac{\mu}{\mu-1} x\right)\right] e^{-r z-(\delta+\lambda) x} \mathrm{~d} x \mathrm{~d} z
$$

which, using $\frac{\partial r}{\partial \lambda}=\frac{\sigma \alpha}{1-\alpha}$, corresponds to the sign of

$$
\int_{0}^{T} \int_{z}^{T}\left[(\delta+\lambda) \frac{\mu T}{\mu-1}+\frac{\mu}{\mu-1}-(\delta+\lambda) \frac{\mu x}{\mu-1}-\frac{(\delta+\lambda) \sigma \alpha}{1-\alpha} z\right] e^{-r z-(\delta+\lambda) x} \mathrm{~d} x \mathrm{~d} z
$$

Since $x \leq T$, the first term of the expression between brackets is bigger than its third term. For the whole term to be positive, it is enough to impose the following condition of the remaining terms, given that $z \leq T: \frac{\mu}{\mu-1} \geq \frac{(\delta+\lambda) \sigma \alpha}{1-\alpha} T$, which gives the condition on $T$ stated in the proposition. Under this condition $\frac{\partial F}{\partial \lambda}>0$. Since $\frac{\partial F}{\partial T}>0$, and $\frac{\partial T}{\partial \lambda}=-\frac{\frac{\partial F}{\partial \lambda}}{\frac{\partial P}{\partial T}}$, we get our result. 
The sufficient condition $T \leq \frac{\kappa}{\delta+\lambda}$ covers by far the usual parameterizations considered in the literature. Indeed, the typical values for $\alpha$ and $\sigma$ imply a parameter $\kappa=\frac{1-\alpha}{\alpha \sigma} \frac{\mu}{\mu-1}$ generally bigger than 1 , and since $\delta+\lambda$ is a relatively small number, our sufficient condition turns out to be far from binding in practice. For example, if the variable operation cost term is quadratic in the efficiency and utilization index $U, \mu=2, \sigma=1$ as in the usual calibrations in macroeconomic models (see Beaudry and Wincoop, 1996, for an econometric justification), and for a capital share $\alpha=\frac{1}{3}$, then our sufficient condition restricts $T$ to be lower than 66 years when $\delta+\lambda=6 \%$, and around 33 years if $\delta+\lambda=12 \%$. This is not restricting at all if one has in mind the average lifetime of private nonresidential equipment and software estimated by BEA for the US economy, which goes from 3 years for software to 33 years for electrical transmission, distribution and industrial apparatus.

Therefore, the capital lifetime $T$ is a decreasing function of the rate of the embodied technical progress for any economically admissible calibration of our model. This deserves two comments. At first, we have to mention that the latter property is indeed consistent with all the recent empirical and theoretical contributions connecting embodied technical change and investment, including the timing of replacement of obsolete goods (see Boucekkine et al., 1998, for a theoretical inspection, and Whelan, 2002, for a more empirical perspective). Second, it seems already clear that the two forms of technical progress have quite distinct economic implications: while the capital lifetime rises when disembodied technical progress accelerates in order to compensate the loss in profitability resulting from the increase in the interest rate, the latter effect is dominated by the increasing efficiency of new vintages under an accelerating embodied technical progress, which on contrary leads to shortening the capital lifetime. The next section highlights more differences concerning use-related depreciation 
and scrapping.

\subsection{The Depreciation Rates}

We shall first state a proposition summarizing the comparative statics of both the use-related depreciation rate and the scrapping rate with respect to the two rates of technical progress, $\gamma$ and $\lambda$. We will comment on these properties just after.

Proposition 6 The use-related depreciation rate $\delta$ is an increasing function of the rate of embodied technical progress $\lambda$ and does not depend on the rate of disembodied technical progress, $\gamma$. The scrapping rate $\xi$ is a decreasing function of $\gamma$. In contrast, it is an increasing function of $\lambda$ if $T \leq \operatorname{Min}\left(\frac{\kappa}{\delta+\lambda}, \frac{X^{0}}{\delta+\lambda+n+g}\right)$, where $\kappa=\frac{1-\alpha}{\alpha \sigma} \frac{\mu}{\mu-1}$ and $X^{0}$ a well-defined strictly positive number depending on the parameters of the model.

Proof: The comparative statics for the use-related depreciation rate are trivial, given equation (27). From (30) it follows that $\xi=H(T, \Psi)=\frac{\Psi}{\mathrm{e}^{\Psi T}-1}, \Psi=\delta+n+\lambda+g$ and

$$
\frac{\partial H}{\partial T}=\frac{-\Psi^{2} e^{(\delta+g) T}}{\left[e^{\Psi T}-1\right]^{2}}<0, \quad \frac{\partial H}{\partial \Psi}=\frac{e^{\Psi T}-1-\Psi T e^{\Psi T}}{\left[e^{\Psi T}-1\right]^{2}}<0 .
$$

$\frac{\partial H}{\partial \Psi}$ is negative since $e^{x}-1-x e^{x}$ is a decreasing function which tends to zero when $x$ tends to 0 and is negative for all $x>0$. Using logarithmic differentiation of $\xi=H(T, \Psi)$, one gets: $\frac{\frac{\partial \xi}{\partial z}}{\xi}=\frac{\frac{\partial \Psi}{\partial z}}{\Psi}-\frac{\frac{\partial\left(e^{\Psi T}-1\right)}{\partial z}}{e^{\Psi T}-1}$, which yields after some algebra: $\frac{\frac{\partial \xi}{\partial z}}{\xi}=\frac{\frac{\partial \Psi}{\partial z}}{\Psi}[1-\Phi(\Psi T)]-\xi e^{\Psi T} \frac{\partial T}{\partial z}$, where $\Phi(X)=\frac{X e^{X}}{e^{X}-1}$, and $z=\lambda, \gamma$. Notice that when $z=\gamma$, we know that the second term is negative by Proposition 4 . Since $\Psi$ is increasing in $\gamma$ and function $\Phi(X)$ is strictly increasing from 1 for $X \geq 0$, it follows that the scrapping rate is a decreasing function of $\gamma$. Things are much more complicated for $\lambda$. Because $\frac{\partial T}{\partial \lambda}<0$ under the conditions of Proposition 4, we have a priori an ambiguous outcome. Notice however that since function $\Phi(X)$ is strictly increasing from 1 , the total effect should be positive, that it is the second term of the logarithmic differentiation should dominate, if $X=\Psi T$ is small enough. This puts another 
upper bound on $T$ : There exists a cut-off value $X^{0}>0$ so that $\xi$ is an increasing function of $\lambda$ if $\Psi T \leq X^{0}$ or $T \leq \frac{X^{0}}{\Psi}$. Then the last part of the proposition follows using Proposition 4.

As in Proposition 4, the property of an increasing scrapping rate with $\lambda$ relies on a sufficient condition on the value of $T$. Although it is less clear here compared to Proposition 4, this condition is again consistent by far with the economically admissible parameterizations of the model. ${ }^{11}$

The use-related depreciation rate and the scrapping rate respond quite differently to technological accelerations. For all the economically admissible parameterizations, both scrapping and use-related depreciation rate increase when the rate of embodied technical change rises: when equipment becomes increasingly efficient, the lifetime of machines is shortened, pushing scrappage upward, and raising the decline rate of utilization of the capital goods, by equation (20), which increases use-related depreciation. However, while the latter does not depend on disembodied technical change, the scrapping rate is shown to fall down when disembodied technical progress accelerates. And this happens because an increase in $\gamma$ leads to lengthen the capital lifetime.

As pointed above, our model has additionally the remarkable property that both rates increase when embodied technical progress accelerate. This has a critical implication for growth accounting: If the total rate of depreciation $(\delta+\xi)$ is not correctly adjusted in such a case, then the growth rate of the capital stock will be over-estimated, which ultimately would deliver a misleading figure for total factor productivity growth, and explain part of

\footnotetext{
${ }^{11}$ We check the sufficient condition for the following wide range of reasonable parameter values: $\alpha=1 / 3$, $\sigma=1, \mu=2, \rho=0.04, n=0.012, \gamma \in[0.05,0.03], \chi \in[0.05,0.12]$, and $\eta \in[0.001,015]$. We also obtain the same results on several alternative parameterizations.
} 
the productivity slowdown puzzle.

\section{Non geometric depreciation schedules}

BEA estimates the depreciation rates of the capital goods using used-asset prices unadjusted for quality. The relative price of a unit of capital of vintage $z$ at time $t$ (a capital good $t-z$ years old) equals the discounted sum of its future returns. In our model:

$$
\widetilde{P}_{z, t}=\int_{t}^{z+T} \mathrm{e}^{-r(s-t)} \eta\left(\mathrm{e}^{-(\delta+\lambda)(s-z)} \mathrm{e}^{(\delta+\lambda) T}-1\right) \mathrm{d} s, \text { for all } z \in(t-T, t]
$$

The relative price of a unit of capital of vintage $t$ at time $t$ (a new capital good) is

$$
\widetilde{P}_{t, t}=\int_{t}^{t+T} \mathrm{e}^{-r(s-t)} \eta\left(\mathrm{e}^{-(\delta+\lambda)(s-t)} \mathrm{e}^{(\delta+\lambda) T}-1\right) \mathrm{d} s .
$$

After some trivial algebra, one can extract the relationship between the relative prices of old and new capital goods,

$$
\widetilde{P}_{z, t}=\mathrm{e}^{-(\delta+\lambda)(t-z)}\left[\widetilde{P}_{t, t}-\eta H(T, r, \lambda, \delta, t-z)\right]
$$

where: ${ }^{12}$

$H(T, r, \lambda, \delta, t-z)=\left(\mathrm{e}^{(\delta+\lambda)(t-z)}-1\right) \int_{t}^{z+T} \mathrm{e}^{-r(s-t)} d s+\int_{z+T}^{t+T} \mathrm{e}^{-r(s-t)}\left(\mathrm{e}^{-(\delta+\lambda)(s-t-T)}-1\right) d s$.

Normalizing $\widetilde{P}_{t, t}=1$, one gets $\widetilde{P}_{z, t}=\mathrm{e}^{-(\delta+\lambda)(t-z)}[1-\eta H(T, r, \lambda, \delta, t-z)]$. We can define a sequence $\left\{\widetilde{\phi}_{s}\right\}_{s=0}^{s=a}$ such that

$$
\widetilde{q}_{a}=\mathrm{e}^{-(\delta+\lambda) a}[1-\eta H(T, r, \lambda, \delta, a)]=\mathrm{e}^{-\int_{0}^{a} \widetilde{\phi}_{s} d s}
$$

where $\widetilde{q}_{a}=\widetilde{P}_{z, t} / \widetilde{P}_{t, t}$ and $a=t-z$, and $\widetilde{\phi}_{a}$ is a function of the rate of embodied technical progress and of the age of the vintage, $\widetilde{\phi}_{a}=\widetilde{\Phi}(\lambda, a)$. $\widetilde{\phi}_{a}$ is the depreciation rate estimated by BEA, which includes the obsolescence rate because BEA uses quality-unadjusted asset

\footnotetext{
${ }^{12}$ Using (37), it is easy to prove that $0<H(T, r, \lambda, \delta, t-z)<\frac{1}{\eta}$ for all $z \in(t-T, t)$.
} 
prices (see Fraumeni,1997). The obsolescence rate is the decline rate of the quality-adjusted relative price of the new capital goods, $\lambda$ in our model. If $\eta>0$ and $T$ is finite, then $\widetilde{\phi}_{a}$ is not constant for all $a$ and the depreciation schedule is not geometric.

As pointed out in Section 2, BEA assumes geometric depreciation schedules for most equipment goods excepting computer equipment and autos. Our analysis suggests that if the lifetime of an asset is large, then the geometric schedule is a good approach because the value of $H(\cdot)$ is not very large, but if the lifetime is short, there might be a marked difference between the true depreciation schedule and the geometric depreciation schedule.

\section{The age-related depreciation rate}

If the relative prices of capital goods are adjusted for quality, then we can calculate the agerelated depreciation rate. If we define the adjusted-quality relative prices as $P_{z, t}=\widetilde{P}_{z, t} \mathrm{e}^{-\lambda z}$ and $P_{t, t}=\widetilde{P}_{t, t} \mathrm{e}^{-\lambda t}$, equation (38) becomes $\frac{P_{z, t}}{P_{t, t}}=\mathrm{e}^{-\delta(t-z)}[1-\eta H(T, r, \lambda, \delta, t-z)]$. We can then define a sequence $\left\{\phi_{s}\right\}_{s=0}^{s=a}$ such that

$$
q_{a}=\mathrm{e}^{-\delta a}[1-\eta H(T, r, \lambda, \delta, a)]=\mathrm{e}^{-\int_{0}^{a} \phi_{s} d s}
$$

where $q_{a}=P_{z, t} / P_{t, t}, a=t-z$, and $\phi_{a}$ is the age-related depreciation rate, which is a function of the rate of embodied technical progress and of the age of the vintage, $\phi_{a}=\Phi(\lambda, a)$. $\phi_{a}$ is a function of $\lambda$ because (i) the lifetime is finite and/or because (ii) utilization of capital declines faster ( $\delta$ is an increasing function of $\lambda$ ). Assuming that $T$ is a decreasing function of $\lambda, \phi_{a}$ is an increasing function of $\lambda$. Therefore, age-related depreciation rate depends on the obsolescence rate in our set-up, and it generally increases as the latter rises.

In the standard neoclassical growth model, the economic depreciation rate of capital is $\Delta=\delta+\lambda$ where $\delta$ is the age-related depreciation rate, which is assumed constant and 
exogenous, and $\lambda$ is the obsolescence rate. Accordingly, this theory would be consistent with the data if the estimated slopes of the relationships between the economic depreciation rates and the decline rates of the corresponding relative prices were close to unity. We have tested this property of the standard neoclassical growth model using the BEA figures for the depreciation rates of equipment and software and their relative prices. Figure 2 is crystal clear: the slope are markedly larger than one, which is much more consistent with the theory of depreciation developed in this paper.

The Geske, Ramey and Shapiro (GRS) finding

GRS estimate the following relationship $\ln q_{a}=-\int_{0}^{a} \phi_{s} d s$ obtaining that the estimated $\phi_{s}$ are high. In a second step, they estimate the modified equation

$$
\ln q_{a}=-\int_{0}^{a}\left(\phi_{s}-\lambda s+\kappa \ln X_{s}\right) d s
$$

where $\kappa \ln X_{a}$ is a proxy of $\lambda a$, and is therefore a proxy of the obsolescence rate, while $X_{a}$ represents a vector of characteristics of the computers of age $a$ and can be consequently viewed as an index of their quality. GRS find that the estimated $\phi_{s}-\lambda$ are near zero for all $s$, which is hardly surprising because $\phi_{s}$ is an increasing function of $\lambda$ as pointed out above. If $\eta=0$, the lifetime of capital is infinite. In such a case, $q_{a}=\mathrm{e}^{-\delta a}$, and the GRS finding is even clearer. The latter relationship can be expressed in logarithms as $\ln q_{a}=-\delta a$. Taking into account that $\kappa \ln X_{a}=\lambda a$, it can be rewritten as

$$
\ln q_{a}=-(\delta-\lambda) a-\kappa\left(\ln X_{t}-\ln X_{z}\right)
$$

If the equation above is to be estimated, then the estimated age-related depreciation rate is necessarily $\widehat{\delta}=\delta-\lambda=\frac{\lambda+\chi}{\mu-1}-\lambda$, which is clearly near zero if $\mu$ close to 2 , and $\chi$ is near zero. Also $\widehat{\kappa}=\lambda$. Our model is therefore fully compatible with the GRS finding. This is far from 
surprising because obsolescence is the main determinant of the depreciation of capital in our set-up.

Capital depreciation and used-asset prices

BEA estimates the depreciation rates using used-asset prices unadjusted for quality and builds the series of capital stocks using these estimates. However, our model show that if the lifetime of capital is finite the decline rate of the used-asset prices is not equal to the depreciation rate of capital. The economic depreciation rate of capital in our model is $\delta+\lambda+\xi$ and the decline rate of the used-asset prices is $\widetilde{\phi}_{a}$ which is defined by (38); it is clear that the economic depreciation rate of capital and the decline rate of the used-asset prices are equal only if the lifetime of capital is infinite. In this case $\xi$ is zero and $\widetilde{\phi}_{a}=\delta+\lambda$ for all $a$. As we have mentioned above, this is due to the fact that when the lifetime of capital is finite, the depreciation resulting from scrapping depends on the amount of capital previously invested, and not only on the price change of a unit of capital.

\section{Conclusions}

In this model, we build a vintage capital model à la Whelan, which incorporates endogenous maintenance costs. In contrast to Whelan, we have a fixed and a variable cost, and more importantly, the variable cost depends on an indicator of the utilization of the vintages. Thanks to this difference, we are able to distinguish between an use-related depreciation rate and a scrapping rate. We characterize the balanced growth paths of the model and put forward many important properties, mostly consistent with the stylized facts. First, the lifetime of capital goods is increasing (resp. decreasing) with the rate of disembodied (resp. embodied) technical progress. Second, the model has the remarkable property that both the 
use-related depreciation and the scrapping rate do rise when embodied technical progress accelerates. In contrast, the latter drops when disembodied technical progress accelerates while the former remains unaffected.

In contrast to the neoclassical growth model in which the age-related depreciation rate is by assumption independent of the obsolescence rate, in our model the former depends on the latter. The age-related depreciation rate increases as the obsolescence accelerates because the lifetime of capital shorts and utilization of capital decline faster. We have tested this implication of our model using the depreciation rates of equipment and software used by BEA and the decline rates of their corresponding relative prices. The observed relationship between these variables is consistent with the theory of depreciation developed in this paper. Dependence of the age-related depreciation rate on the obsolescence rate also allows to explain the empirical findings recently put forward by Geske, Ramey and Shapiro.

Last we point out some important implications of finite lived capital goods. BEA assumes geometric depreciation schedules for most of capital goods. However, our analysis implies that as embodied technical progress accelerates, the assumption of a constant depreciation rate becomes less appropriate because the lifetime of capital gets shorter in such a circumstance. Moreover, if the lifetime of capital is finite, the depreciation rate of capital is no longer equal to the price change of the capital goods because the scrapping depreciation rate depends on the amount of scrapped capital, and not only on the price change of a unit of capital. 


\section{References}

Beaudry, P. and E. Van Wincoop (1996), "The Intertemporal Elasticity of Substitution: An Exploration Using a US Panel of State Data" Economica 63, 495-512.

Bischoff, C.W. and E.C. Kokkelenberg (1987), "Capacity Utilization and Depreciation in Use", Applied Economics 19, 995-1007.

Boucekkine, R. and R. Ruiz-Tamarit (2003), "Capital Maintenance and Investment: Complements or Substitutes?", Journal of Economics 78, 1-28.

Boucekkine, R., M. Germain, O. Licandro and A. Magnus (1998), "Creative Destruction, Investment Volatility and the Average Age of Capital", Journal of Economic Growth 3, 361-384.

Bureau of Economic Analysis (2003), Fixed Assets and Consumer Durable Goods in the United States, 1925-99. Washington, DC: U.S. Government Printing Office

Burnside, C. and M. Eichenbaum (1996), "Factor-Hoarding and the Propagation of BusinessCycle Shocks", American Economic Review 86 (5), 1154-1174.

Epstein, L. and M. Denny (1980), "Endogenous Capital Utilization in a Short-Run Production Model", Journal of Econometrics 12, 189-207.

Feldstein, M. and M. Rotschild (1974), "Towards an Economic Theory of Replacement Investment", Econometrica 42, 393-423.

Fraumeni, B. M. (1997), "The Measurement of Depreciation in the U.S. National Income and Products Accounts", Survey of Current Business Bureau of Economic Analysis, July, $7-23$. 
Geske, M., V. Ramey and M. Shapiro (2004), "Why Do Computers Depreciate?", NBER Working Paper 10831.

Greenwood, J., Hercowitz, Z. and Krusell, P. (1997), Long-Run Implications of InvestmentSpecific Technological Change, American Economic Review 87, 342-362.

Greenwood, J., Z. Hercowitz and G. Huffman (1988), "Investment, Capital Utilization and the real Business Cycle", American Economic Review 78, 402-417.

Griliches, Z. (1960), "Measuring Inputs in Agriculture: A Critical Survey", Journal of Farm Economics XLII, 1411-1427.

Gylafson, T. and Zoega (2001), "Obsolescence", Discussion Paper 2833, CEPR (London).

Hamilton, B. and M. Macauley (1980), "Competition and Car Longevity", Discussion Paper 98/20, Johns Hopkins University, Washington.

McGrattan, E. and J.A. Schmitz (1999), "Maintenance and Repair: Too Big to Ignore", Quarterly Review, 23, 4, Federal Reserve Bank of Minneapolis.

Musso, P. (2004), "Productivity Slowdown and Resurgence: The Role of Capital Obsolescence", Working Paper 2004-1, GREDEG, Nice.

Nickell, S. (1975), "A Closer Look at Replacement Investment", Journal of Economic Theory $10,54-88$.

Whelan, K. (2002), "Computers, Obsolescence, and Productivity", The Review of Economics and Statistics 84, 445-461. 
TABLE 1: Depreciation rate, service lifetime (years) and decline rate of the relative price (annual average 1959-2003) of equipment and software by types

Category

Computers and peripheral equipment

Software

Communication equipment

Medical equipment and instruments

Photocopy and related equipment

Office and accounting equipment

Fabricated metal products

Engines and turbines

Metalworking machinery

Special industry machinery

General industrial, equipment

Electrical transm., industrial apparatus

Trucks, buses and truck trailers

Autos

Aircraft

Ships and boats

Railroad equipment

Furniture and fixtures

Agricultural and machinery

Construction machinery

Mining and oilfield machinery

Service industry machinery

Electrical equipment

Other
Depreciation rate

Service lifetime

0.4

0.13

0.135

0.18

0.312

0.092

0.129

0.18

0.103

0.107

0.05

0.163

0.28

0.096

0.061

0.059

0.138

0.118

0.155

0.15

0.158

0.183

0.147
4.33

13

12

9

7

18

20

16

16

16

33

16.5

10

17.5

27

28

12

14

10

11

10.5

9

11
Decline rate

0.203

0.049

0.028

0.012

0.036

0.031

0.006

0.001

$-0.001$

$-0.003$

0.002

0.014

0.007

0.024

$-0.002$

$-0.001$

0.003

0.006

$-0.002$

$-0.004$

$-0.004$

0.010

0.018

0.010

Source: Department of Commerce, Bureau of Economic Analysis. 


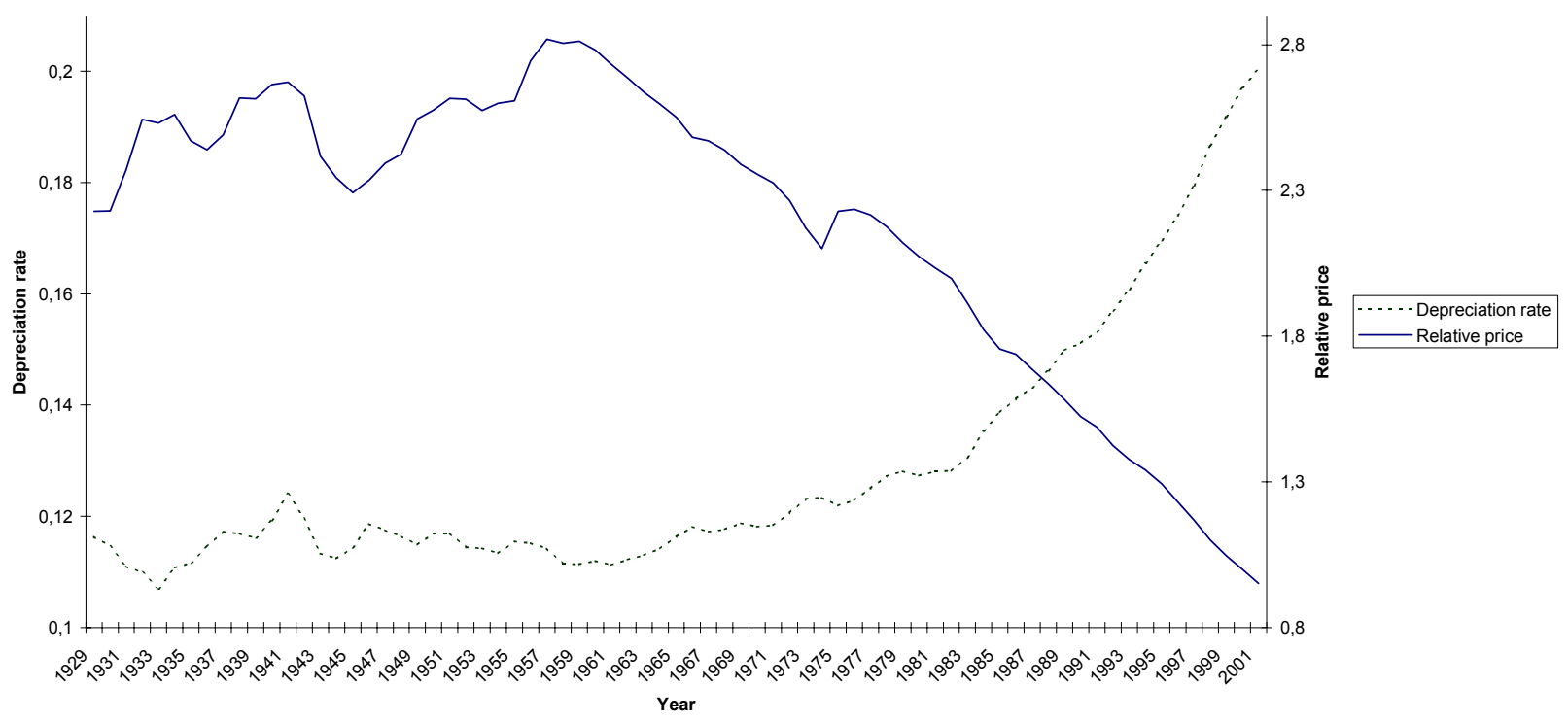

Figure 1: Depreciation rate and relative price of private nonresidential equipment and software, 1929-2001

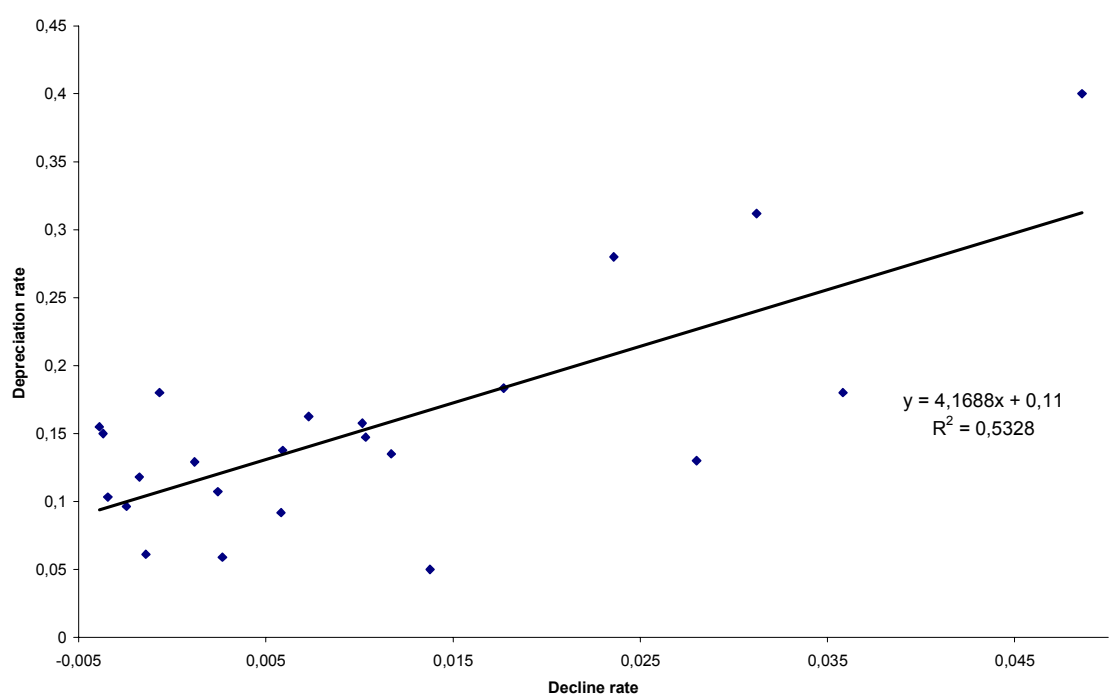

Figure 2: Depreciation rate and decline rate of the relative price of private nonresidential equipment by types 


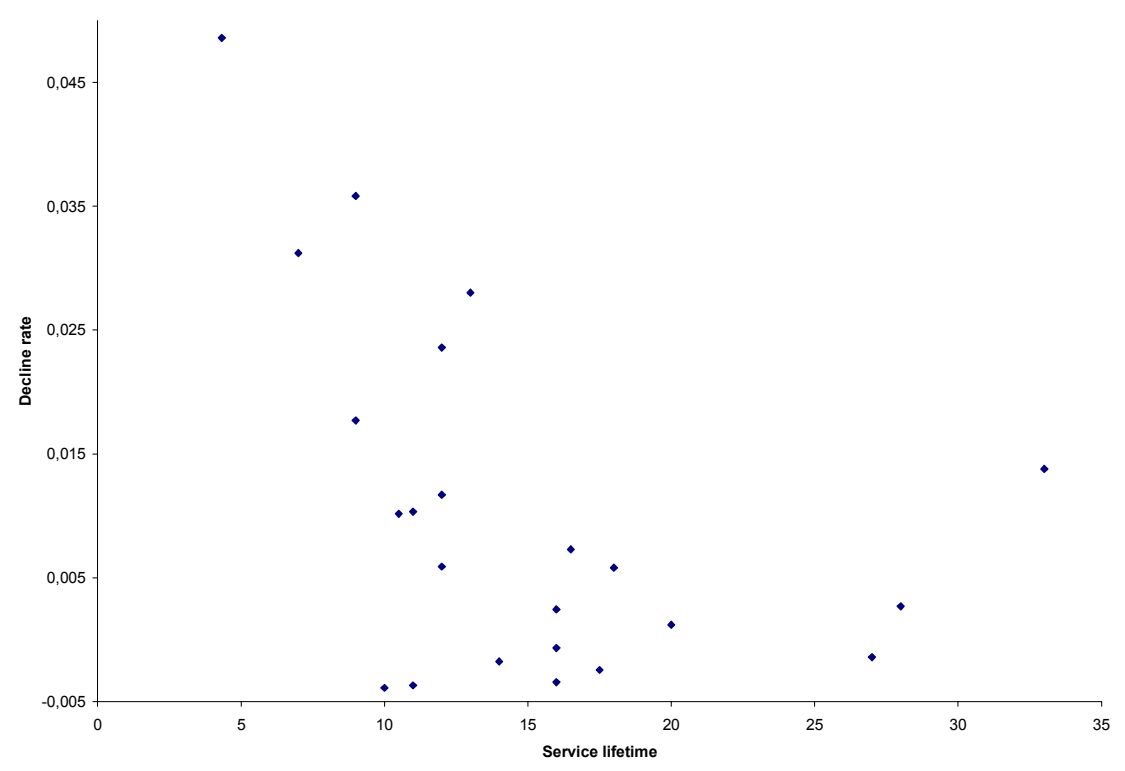

Figure 3: Service lifetime and decline rate of the relative price of private equipment and software by types 
Département des Sciences Économiques de l'Université catholique de Louvain

Institut de Recherches Économiques et Sociales

Place Montesquieu, 3

1348 Louvain-la-Neuve, Belgique 